\title{
Image Matching Using Distance Transform
}

\author{
Abdul Ghafoor ${ }^{1}$, Rao Naveed Iqbal ${ }^{2}$, and Shoab Khan ${ }^{2}$ \\ ${ }^{1}$ Department of Electrical Engineering, College of Electrical and Mechanical Engineering, \\ National University of Sciences and Technology, \\ Rawalpindi, Pakistan \\ a ghafoor30@yahoo.com \\ ${ }^{2}$ Department of Computer Engineering, College of Electrical and Mechanical Engineering, \\ National University of Sciences and Technology, \\ Rawalpindi, Pakistan \\ \{rao_naveed,kshoab\}@yahoo.com
}

\begin{abstract}
Image matching is an important task. There are many available methods for occluded image matching, e.g. Hausdorff distance, Distance Transformi and wavelet decomposition based methods. We proposed Modified Chamfer Matching Algorithm (MCMA) [16], a new simple distance transform based image matching algorithm. Here we propose few more matching measures and compare them. Basic concepts relating to distance transform are also explained. Examples to demonstrate the algorithm and necessary results are also included.
\end{abstract}

\section{Introduction}

Image matching is a central problem in the pattern recognition, image analysis, robotics and computer vision. The exact position of objects, units and features related to image must be known. The noise and errors in preprocessing of images (e.g., segmentation and edge detection) aggravates the matching problems. The parameters like translation, rotation and scaling must often be taken into account in most of the image matching algorithms. Moreover, the input to different sensors at the same time or input to same sensor at different times will always result in occluded output, and matching an occluded image is always been a challenging task.

Based on the level of image feature extraction, the matching methods developed in the past can be divided into three classes: algorithms that use image pixel values directly, e.g., correlation methods; algorithms that uses low level features such as edges and corners e.g., distance transform and Hausdorff distance based ; and algorithms that uses high level features such as identified objects or relation between the features, e.g., graph-theoretic-methods. The methods making use of image pixel value directly are sensitive to changes between images. High level matching methods are very insensitive to these disturbances. But, the disadvantage is, the high level features must first be extracted and identified and that is, in most cases a difficult problem.

Chamfer (distance transform) matching [1] is a technique for finding the best fit of edge points from two different images, by minimizing a generalized distance between 
them. The edge points of one image are transformed by a set of parametric transformation equations that describes how the image can be geometrically distorted in relation to one another. The original idea has several good properties, e.g., ability to handle imperfect data but also had false matching. Measure of correspondence between patterns to be matched has been improved to have fewer false matches [2]. As the matching problem is computation intensive, a multi resolution approach [3], hierarchical chamfer matching algorithm (HCMA) [4], to speed up the computation considerably, so much so that matching problem that could not be solved with single resolution algorithm (as computational load was too heavy), could be solved using moderate computation time. Other known robust matching methods are Hausdorff distance [5], its variants [6]-[12], and wavelet decomposition method [13].

We proposed an image matching algorithm [16] using distance transform which is to an extant different from conventional chamfer matching [1]-[4]. It is low level feature based method, in which edge points or low level feature points are extracted from digital images (using any suitable edge extraction scheme), converted to binary images, which are distance transformed, and then distance transform is used for matching. The distance transform of template is superimposed on the distance transform of the model and values are subtracted pixel wise and minimum RMS value gives the matching position.

In this paper we propose few more matching measures and compare there results. Proposed matching measures are: ranked highest numbers of zeros, range, and minimum average in addition to RMS value (already proposed [16]).

This paper is divided into five sections including this section. In section- 2 the basic concept regarding distance transform are discussed. MCMA with additional proposed measures is explained in section-3, simulation results are given in section-4 and finally conclusion in section-5.

\section{Distance Transform}

Two binary images based on feature and non-feature points, are to be matched. The feature can be any well defined object visible in both images. The distance transform of the image is formed by assigning each non-edge pixel a value that is a measure of distance the nearest edge pixel. The true Euclidian distance [14] is resource demanding (time, memory) to compute, therefore an approximation is used. Good integer approximations of Euclidean distance can be computed by a process known as chamfer 3-4 distance [2] and [4]. The process of converting a binary image to an approximate distance image is called distance transformation (DT). In the binary edge image each edge pixel is first set to zero and each non edge pixel is set to infinity. If the DT is computed by parallel propagation of local distances then at each iteration each pixel obtains a new value using the expression:

$$
\begin{aligned}
v_{\mathrm{i}, \mathrm{j}}^{\mathrm{k}} & =\underset{\mathrm{minimum}}{\left.1_{\mathrm{i}, \mathrm{j}+1}+3, v^{\mathrm{k}-1}{ }_{\mathrm{i}-1, \mathrm{j}-1}+4, v^{\mathrm{k}-1}{ }_{\mathrm{i}-1, \mathrm{j}}+3,4, v^{\mathrm{k}-1}{ }_{\mathrm{i}+1, \mathrm{j}}+3, v^{\mathrm{k}-1}{ }_{\mathrm{i}+1, \mathrm{j}+1}+4\right)}+4, v^{\mathrm{k}-1}{ }_{\mathrm{i}, \mathrm{j}-1}+3, v^{\mathrm{k}-1}{ }_{\mathrm{i}, \mathrm{j}}, v^{\mathrm{k}-}
\end{aligned}
$$


where $v^{\mathrm{k}} \mathrm{i}, \mathrm{j}$ is the value of the pixel in position $(\mathrm{i}, \mathrm{j})$ at iteration $\mathrm{k}$. The iteration continues until no value changes. The number of iterations is proportional to the longest distance occurring in the image.

\section{Proposed Scheme}

We proposed Modified Chamfer Matching Algorithm [16] where we proposed only one matching measure. Here we propose a few more matching measures for MCMA.

\subsection{MCMA}

MCMA is low level feature based method. In MCMA edge points or low level feature points are extracted from digital images (using any suitable edge extraction scheme), converted to binary images, which are distance transformed, and then distance transform is used for matching. The distance transform of template is superimposed on the distance transform of the model and values are subtracted pixel wise and matching is found as per the matching measure and translational parameter. We proposed RMS matching measure.

\subsection{Algorithm}

Consider a reference image $\mathrm{A}$ of size $\mathrm{MxN}$ and a template image $\mathrm{B}$ of size $\mathrm{OxP}$ with elements $a_{m, n}$ and $b_{o, p}$ respectively. Assume $A \geq B ; m=1,2, \ldots, M ; n=1,2, \ldots, N$; $\mathrm{o}=1,2, \ldots, \mathrm{O} ; \mathrm{p}=1,2, \ldots, \mathrm{P} ;$ range $1<$ range 2 . We can define $\mathrm{C}$, such that $\mathrm{C}=\mathrm{o \epsilon} \mathrm{O}, \mathrm{p} \in \mathrm{P}$ $\left[\mathrm{c}_{\mathrm{o}, \mathrm{p}}\right]$ for all $\mathrm{o}$ and $\mathrm{p}$.

for $\mathrm{q}=0: \mathrm{m}-\mathrm{o}$

for $r=0: n-p$

$$
\mathrm{C}=\mathrm{o \epsilon} \mathrm{O}, \mathrm{p} \epsilon \mathrm{P}\left[\left[\mathrm{a}_{\mathrm{o}+\mathrm{q}, \mathrm{p}+\mathrm{r}}\right]-\mathrm{B}\right]
$$

end

$$
\text { Measure }=\text { rms average }\left[o € \mathrm{O}, \mathrm{p} \epsilon \mathrm{P}\left[\mathrm{c}_{\mathrm{o}, \mathrm{p}}\right]\right]
$$

end

\subsection{Proposed Matching Measures}

Here we define three more matching measures:

$$
\begin{aligned}
& \text { Measure } 1=\text { find }\left[\mathrm{o} \epsilon \mathrm{O}, \mathrm{p} \in \mathrm{P}\left[\mathrm{c}_{\mathrm{o}, \mathrm{p}}==0\right]\right] \\
& \text { Measure } 2=\text { find }\left[\mathrm{o} \Theta \mathrm{O}, \mathrm{p} \in \mathrm{P}\left[\text { range } 1<\mathrm{c}_{\mathrm{o}, \mathrm{p}}<\text { range } 2\right]\right]
\end{aligned}
$$

OR Measure $2=$ find $\left[\mathrm{c}_{\mathrm{o}, \mathrm{p}} \mid<\right.$ range $]$

$$
\text { Measure } 3=\text { arithmetic average }\left[o € O, p \in P\left[c_{o, p}\right]\right]
$$




\subsection{Image Matching using Matching Measures}

The DT of template is subtracted from DT of reference and using suitable measure, object is matched. Although we propose four matching measures but any one can be used for matching.

The first matching measure searches the maximum number of zero elements after subtracting the template DT from reference DT. If the number of zero elements are equal to the maximum size of the template, then there is a perfect match. However if the number of zero elements after subtraction is not equal to the maximum array size, the match is not the perfect and that will happen in case of occluded, rotated, scaled and perturbed images.

In the second matching measure, after subtraction of template from reference, the resultant image points are checked for the number of pixels within predefined range, and number is maximized for matching. To avoid false matching, range is reduced. Goal is to find a translation at which the numbers in the range maximizes, which will be a match and we can also give the confidence level of matching.

In the third matching measure, we propose taking the arithmetic average (2) of all the pixels, and find the absolute minimum value. The minimum value is the match, and we can have a confidence of matching level. If absolute minimum value is zero at a specific translation we have perfect match.

In the fourth matching measure [16], we propose taking the RMS average (3) of all the numbers and find the minimum value. The minimum value is the match. If minimum RMS value is zero at a specific translation we have perfect match.

\section{Experimental Results}

In this section we present the experimental results for evaluating the efficiency of the proposed matching algorithm. All experiments were implemented on a personal computer with Pentium IV, $1.7 \mathrm{GHz}$ processor. The images of different sizes having 8 bit gray level were used. All the images (reference/template) were applied soble operator for edge detection. After finding the DT image, templates were matched to the reference as per the matching measures. Results were simulated in Matlab 6.1.

Fig. 4.1 [15] is a $252 \times 238,8$ bit gray level reference image. Fig. 4.2 [15] is a $83 \times 79,8$ bit gray level template image. Template is rotated and perturbed with noise. Soble operator was used for edge detection. These edge images are then converted to 3-4 DT images, applied as input to proposed algorithm, and translation parameters are found as per matching measure. RMS average matching measure and range matching measures were used in this example. Range defined for this example is $-6<$ range $<+6$. It took about 26 seconds for producing the match including about 14 seconds DT computation time. For real time matching, DT of reference can be computed off shelf, and about $50 \%$ computation time could be saved. Fig. 4.3 shows that template is matched to reference. Only translational parameter is used for matching. A rotated image was perfectly matched with about $98 \%$ confidence level using RMS matching measure and $40 \%$ confidence level using range matching measure. If we increase the range, confidence level will increase, but range can be increased to an extant for 
avoiding false match. Fig 4.4 and Fig 4.5 shows matching graphs of RMS and range measure, respectively at the translation $(121,166)$. For this example highest number of zeros matching measure will not provide correct results because of template rotation.

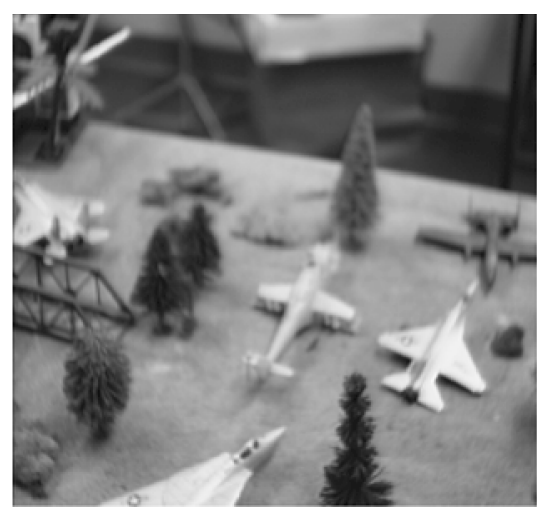

Fig. $4.1252 \times 238,8$ bit gray level reference image

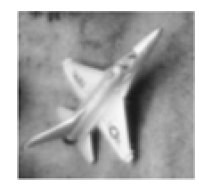

Fig. 4.2 $83 \times 79,8$ bit gray level template image

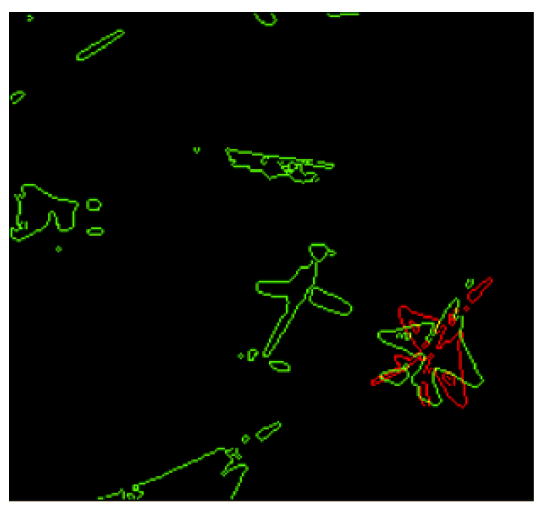

Fig. $4.3 \quad$ Match 


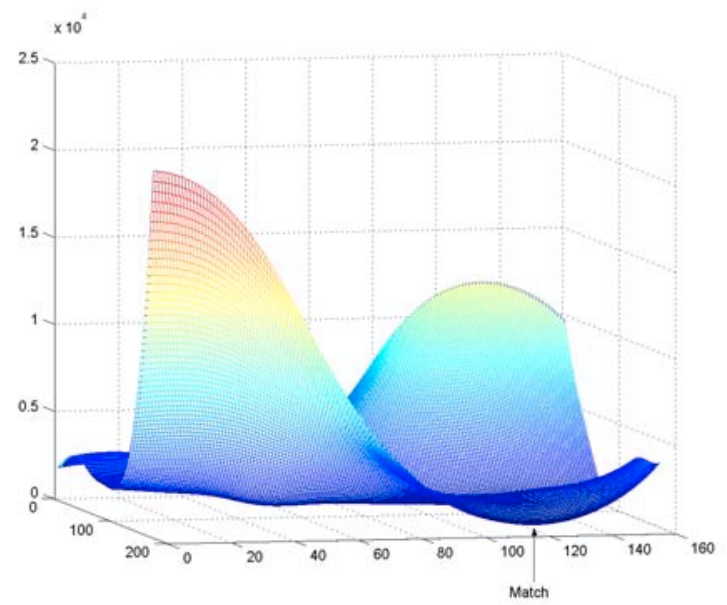

Fig. 4.4 3D view of RMS values at every translation

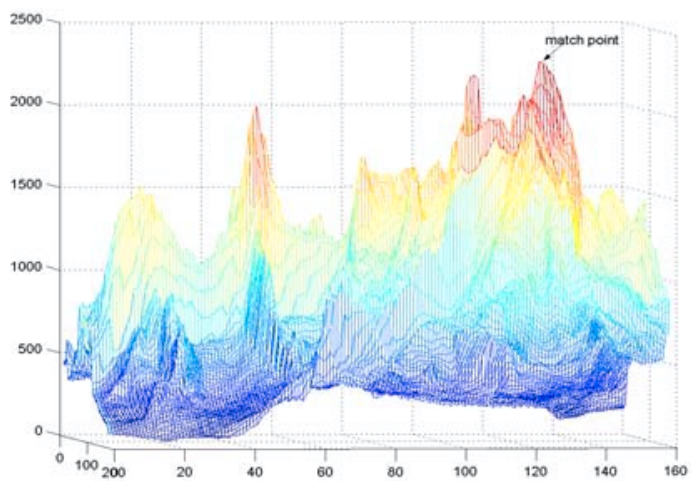

Fig. 4.5 Number of pixels in the range (-6 to 6) for each translation

\section{Conclusions}

In this paper we proposed an extension to the existing modified chamfer matching algorithm. We discussed a few new matching measures for modified chamfer matching algorithm. One experimental example is presented using different matching measures. Except highest number of zeros matching measure, experimental results prove the proposed method, a time efficient, robust, rotation and scale invariant. 


\section{References}

1. H.G. Barrow, J.M. Tanenbaum, R.C. Botles and H. C. Wolf, "Parametric correspondence and chamfer matching: Two new techniques for image matching," in Proceedings of $5^{\text {th }}$ International Joint Conference on Artificial Intelligence, Cambridge, MA, pp. 659$653,1977$.

2. G. Borgefores, "An improved version of the chamfer matching algorithm," in $7^{\text {th }}$ Int. Conf, Pattern Recognition, Montreal, P.Q.,Canada, pp. 1175-1177, 1984.

3. A. Rosenfeld, "Multi resolution image representation", in Digital Image Analysis, S. Levialdi, Ed., London: Pitman, pp. 18--28, 1984.

4. Gunnila Borgefores, "Hierarchical chamfer matching: A parametric edge matching algorithm", IEEE transaction on Pattern Analysis and Machine Intelligence. Vol. 10, no. 6, pp. 849--856, 1988.

5. Daniel P. Huttenlocher, Gregory A. Klanderman, and William J. Ruklidge, "Comparing images using the Hausdorff distance", IEEE Trans. Patt. Anal. Machine Intell., vol 15, no 9, pp 850--863,1993.

6. D.P. Huttenlocher, K. Kedem, M. Sharir, “ The upper envelope of Voronoi surfaces and its applications, Discrete Comput. Geom. 9, pp 267-291, 1993.

7. L.Paul Chew, Kalara Kedem, "Getting around a lower bound for the minimum Hausdorff distance”, Computational Geometry 10, pp197--202, 1998.

8. J.You, E.Pissaloux , J-L. Hellec, and P. Bonnin, "A guided image matching approach using hausdorff distance with intresting points detection", IEEE, pp 968--972, 1994

9. B.B. Chaudhuri and A. Rosenfeld, "A modified Hausdorff distance between fuzzy sets", Information Sciences 118, pp 159-171,1999.

10.David M. Mount, Nathan S. Netanyahu, Jacqueline Le Moigne, "Efficient algorithms for robust feature matching", Pattern Recognition 32 pp 17--38, 1999.

11.Oh-Kyu Kwon, Dong-Gyu Sim, Rae-Hong Park, "Robust Hausdorff distance matching algorithms using pyramidal structures”, Pattern Recognition 34, pp 2005-2013, 2001.

12.Yongsheng Gao, Maylor K.H. Leung, "Line segment Hausdorff distance on face matching”, Pattern Recognition 35, pp 361-371, 2002.

13.D. M. Tsai ,C. H. Chiang, “ Rotation invariant pattern matching using wavelet decomposition", Pattern Recognition Letters 23, pp 191-201, 2002.

14. S. Hezel, A. Kugel, R. Manner, D.M. Gavrila, "FPGA-based Template Matching using Distance Transforms", Proceedings 10th Annual IEEE Symposium on Field-Programmable Custom Computing Machines, 2002.

15.http://www-cgrl.cs.mcgill.ca/ godfried/teaching/cg-projects/98/normand/main.html

16.Abdul Ghafoor, Rao Naveed Iqbal, and Shoab A. Khan, "Modified Chamfer Matching Algorithm" in proceeding Springer, IDEAL, Honk Kong, March 2003. 